\author{
EVS26 \\ Los Angeles, California, May 6-9, 2012
}

\title{
Condition Monitoring Sensor for Electric Vehicle Motor and Generator Insulation Systems
}

\author{
Ken Watkins \\ Polymer Aging Concepts, Inc., 79 Turner Rd, Dahlonega, GA 30533 \\ kwatkins@PolymerAgingConcepts.com \\ C. P. Wong \\ School of Materials Science and Engineering, Georgia Institute of Technology, Atlanta, GA, \\ cp.wong@mse.gatech.
}

\begin{abstract}
Electric vehicle (EV) motors are subject to extreme and variable loads, resulting in degradation of winding insulation due to high temperatures. This paper describes research on a new conductive composite sensor, which uses insulation resin as a matrix of the sensor element. The sensor, embedded in the windings of EV motors, will provide data on remaining design life of the insulation based on actual vehicle operational and environmental conditions. Improved condition monitoring of the insulation systems of high-performance EV motors can reduce in-use failures by identifying prematurely degraded insulation systems, and providing data for quality improvement programs.
\end{abstract}

Keywords: Electric vehicle condition monitoring, electric motor condition monitoring, electric insulation condition monitoring, electric motor insulation

\section{Introduction}

Electric/hybrid vehicle motors are especially susceptible to aging effects due to the high motor loads encountered in steep terrains, high payload and high ambient temperatures. Motor drive systems for electric vehicles are much higher power density than typical applications. [1] Failure of the insulation system of these components can lead to vehicle breakdowns that could be prevented if the condition of the insulation system is known and replaced before motor failure.

New diagnostic methods are being looked at such as those analyzing mechanical and electromagnetic deviations in permanent magnet motors used in automotive powertrains [2]. While these methods are very useful, they may be complex, expensive and, even when monitored continuously, some insulation failures occur with no warning.

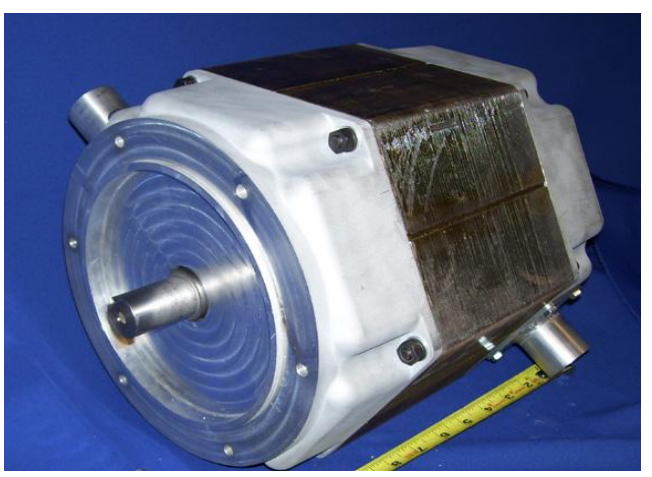

Figure 1: Electric vehicle motors and generators are subject to extreme load variations and variable ambient conditions, putting high stress on winding insulation systems. 
The degradation mechanisms of modern insulation systems are complex and are highly dependent on the actual environmental and operational conditions of the component. Current methods to measure insulation system degradation are often complex, expensive and require specialized training to interpret.

This paper summarizes the results of research conducted on condition monitoring sensors for high performance epoxy and polyester insulation systems in nuclear power plant electrical equipment. This work is also applicable to electric vehicles and other high performance motors and generators. The research was conducted by Polymer Aging Concepts, Inc., and the School of Materials Science and Engineering at the Georgia Institute of Technology. [3, 4]

Composite degradation sensors are passive, meaning that they respond to aging effects of the insulation automatically, requiring electrical power only when interrogated. Further development will demonstrate suitability of conductive composite degradation sensors for intelligent passive radio frequency identification devices (RFIDs), periodic monitoring by simple resistance measuring devices, or real-time, in-situ monitoring of insulation as part of a comprehensive condition monitoring system.

\section{DESCRIPTION OF THE ACTUAL WORK}

The condition monitoring sensors of this research are conductive composites made, in part, of a tiny portion of the same insulation as that used in electric motors and conductive fillers. The sensors are packaged and embedded within the stator windings of the motor so the sensor "sees" the same environment as the insulation itself. During motor aging under thermal and other environmental conditions, degradation mechanisms such as cross-linking, chain scission, and loss of volatiles result in shrinkage of the insulation and the insulation portion of the sensor. Since the conductive filler portion of the sensor remains constant, the volume fraction of the conductive filler increases and the resistance of the sensor decreases. Minute changes in the insulation shrinkage during aging provide large changes on the sensors resistance.
The figure below shows the effect of the volume fraction of the conductive filler on resistivity of the sensor. As the sensor "ages," the matrix (insulation) shrinks, increasing the volume fraction of the conductive filler. As the volume fraction of the conductive filler of the sensor increases, the resistivity decreases as the sensor continues to age.

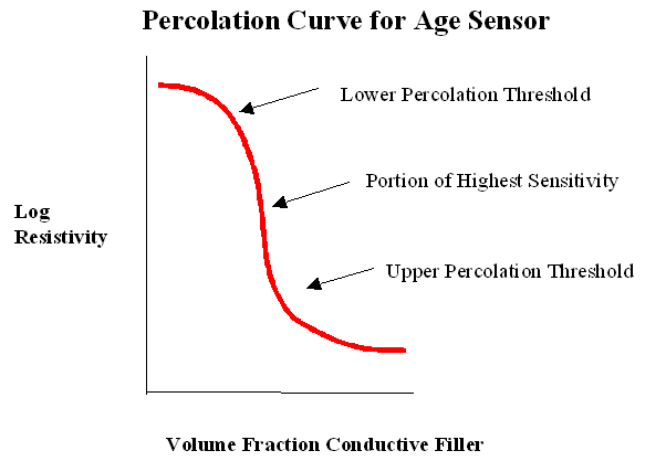

Figure 2: Electrical percolation curve showing the effect of increasing the volume fraction of the conductive filler due to aging.

The photograph below shows insulation sensors made from a commercial epoxy resin used in high performance stator insulation systems. Carbon black is used as the conductive filler. Electrodes, embedded in the composite sensor element, provide a means to measure resistance of the sensor element as the insulation degrades under thermo-oxidative conditions.

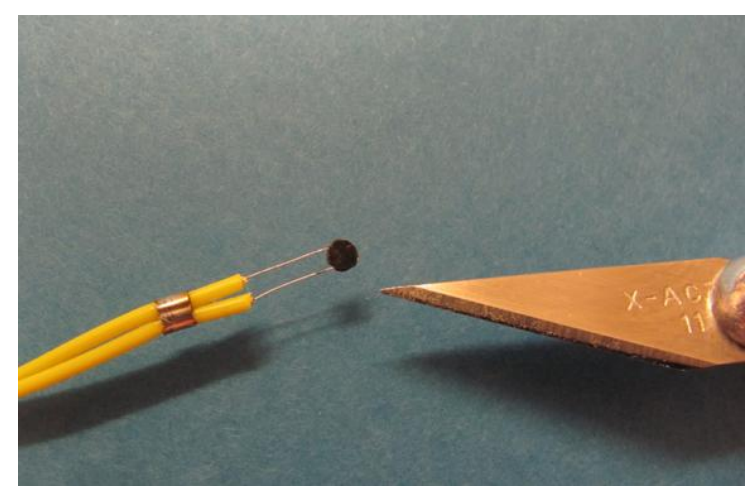

Figure 3: Conductive composite sensor element made from a motor insulation epoxy resin and carbon black conductive filler, change resistance as the epoxy insulation shrinks during thermaloxidative aging.

The graph below shows sensor resistance of the sensor element of Figure 3 during thermaloxidative aging at multiple temperatures. The rate of resistance change increases with temperature. 


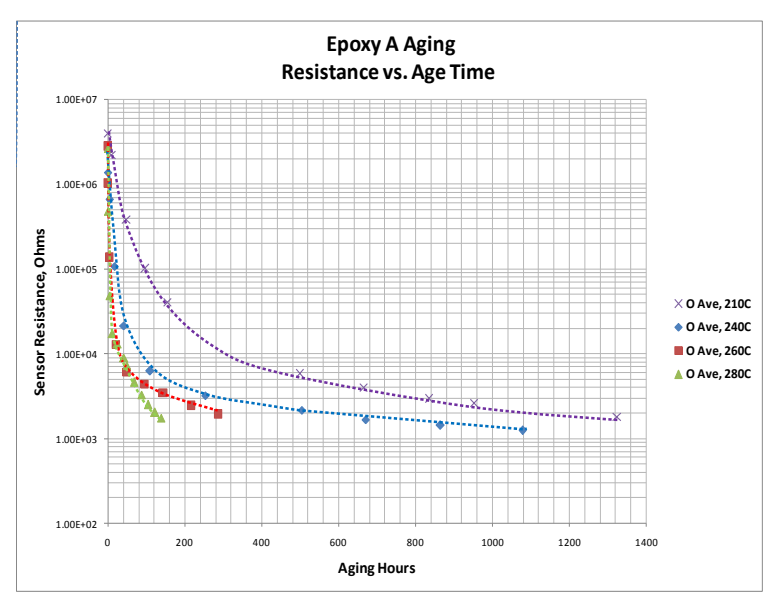

Figure 4: Effect of aging temperature on epoxy insulation sensor resistance. Note significantly faster rate of resistance change with higher temperatures.

The figure below shows the Arrhenius behavior of epoxy insulation sensors based on the time for resistance to decrease to a selected value at multiple temperatures. The graph also shows thermal endurance data (destructive testing of winding formettes) at multiple temperatures for a 6000 horsepower motor. Good Arrhenius behavior of the sensors allows modeling of the target insulation from sensor resistance data.

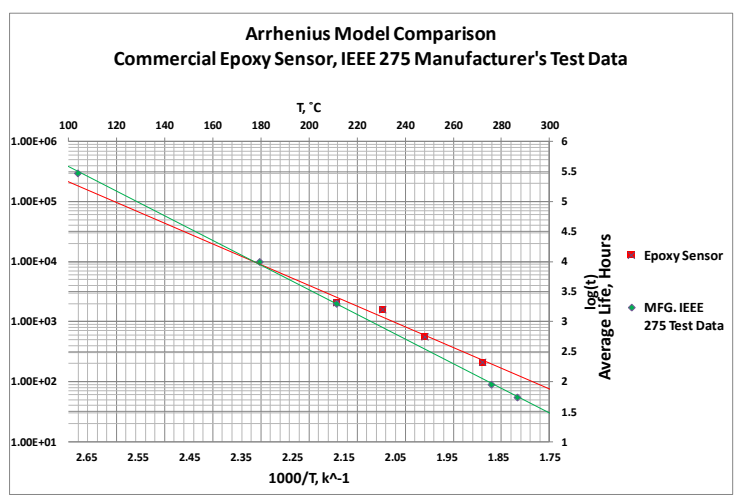

Figure 5: Arrhenius graph of destructive thermal endurance test data for a motor insulation and epoxy sensor degradation data from resistance measurements. Good Arrhenius behaviour of the sensor data allows accurate modelling of insulation degradation.

The figure below shows packaged insulation sensors. The packages allow "breathing" so that the sensor element "sees" the same environment as the insulation itself.

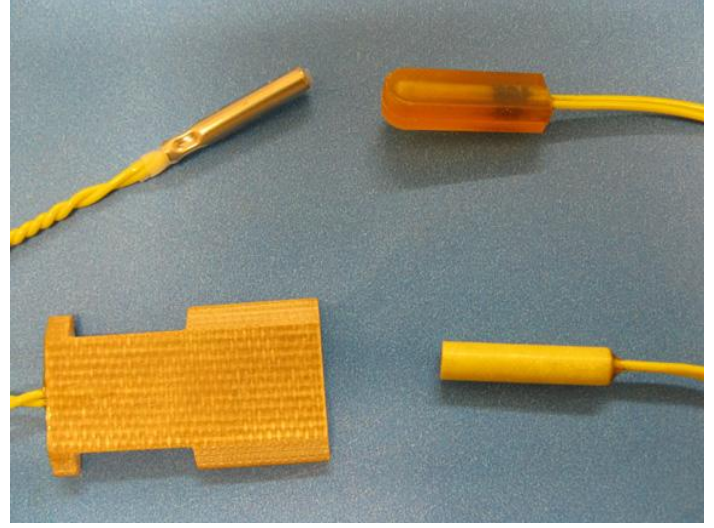

Figure 6: Packaged insulation sensors for installation in electrical motors and generators. The packages provide "breathing" for the sensor elements so that they "see" the same environment as the insulation.

The photograph below shows an insulation sensor installed in a fractional horsepower electric motor for testing. The sensor is attached to the end windings so that the sensor experiences a similar temperature and atmospheric environment as the insulation itself.

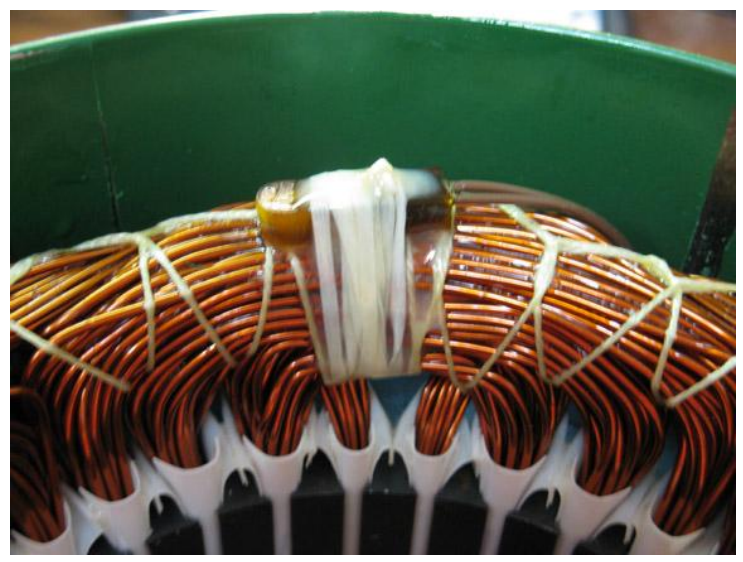

Figure 7: Insulation sensor attached to the end windings of a fractional horsepower electric motor.

The sensor experiences a similar thermal and atmospheric environment as the winding insulation.

The photograph below shows the sensor of Figure 7, connected to test points on a motor, being measured by a field ohmmeter. A conversion chart, attached to the motor converts resistance readings to percent remaining design life of the insulation based on actual motor operation and environmental conditions. In other applications, the insulation sensor can be connected to an existing wired or wireless condition monitoring system by use of resistance-to-remaining life conversion algorithms. 


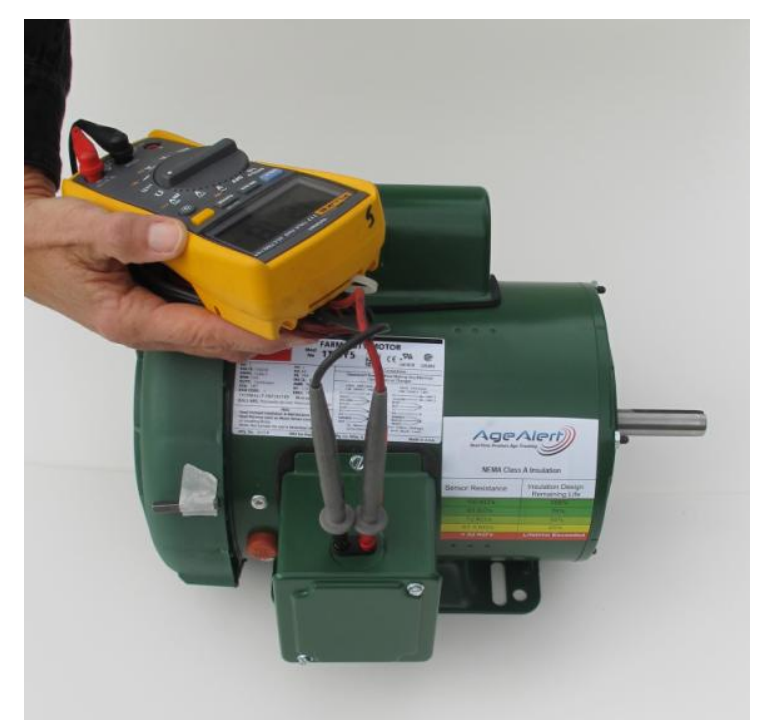

Figure 8: A conversion chart on the motor converts sensor resistance to percent remaining design life of the insulation based on actual motor environment and operating history. Alternatively, the sensor connects to wired or wireless condition monitoring systems.

Current beta prototype sensors are being tested in NEMA class $B, F$ and $H$ motors. Sensor resistance will be measured and used in projected lifetime analysis. Installation in a 6,000 horsepower motor has demonstrated high sensitivity of this condition monitoring technology by showing a few hours of insulation aging at normal operating temperature.

In addition to the "passive" (no electrical power until interrogated) feature of conductive composite sensors, they inherently "integrate" degradation so that sensor resistance always corresponds to total current aging without data logging or environmental monitoring.

\section{Conclusions}

Trials and Arrhenius behaviour of conductive composite sensor resistance demonstrate feasibility of conductive composite degradation sensors to model insulation degradation under thermo-oxidative conditions. Installation and test of sensors in an operating motor demonstrate high sensitivity to aging of this methodology by the ability to monitor several hours of insulation aging with an ohmmeter without special training or equipment.
Additional testing will be performed under multiple environments (chemical, humidity, salt spray) to determine the ability of conductive composite sensors to monitor degradation under complex, real-world environments. Conductive composite sensors, embedded in the windings of electric vehicle motors and generators, will give advance warning of significant insulation degradation resulting from actual vehicle operational and environmental conditions. Sensor data can be connected to an electric vehicle "check electric motor" or "check electric generator" light.

These sensors will also provide objective aging data which provide vehicle manufacturers a basis for further improvements in vehicle safety, reliability and cost-effective maintenance cycles.

\section{Acknowledgments}

The Authors express appreciation to the U. S. Department of Energy, Office of Nuclear Energy for financial assistance during this project. Special thanks to Dr. Madeline Feltus, Department of Energy Technical Advisor, for technical consultation during the project.

\section{References}

[[1] Wen, Xuhui, et al.: "Lifetime Model Research of Motor Drive System for Electric Vehicles", Proceeding of International Conference on Electrical Machines and Systems, Seoul, Korea, (2007).

[2] Flach, Andreia, Draeger, Florian, Ayeb, Mohamed, Brabetz, Ludwig: "A New Approach to Diagnostics for Permanent-Magnet Motors in Automotive Powertrain Systems", IEEE 978-14244-9303, June, 2011.

[3] Y. Sun, L. Fan, K. Watkins, J. Peak, C. P. Wong. An Electrical Approach to Monitor Wire and Cable Thermal Oxidation Aging Condition Based on Carbon Black Filled Conductive Polymer Composite. 2, Atlanta, GA : Journal of Applied Polymer and Science, 2004, Vol. 93.

[4] Watkins, Kenneth S., Jr.: "Method and Apparatus for Measuring Degradation of Insulation of Electrical Power System Devices", U. S. Patent No. 7,659, 728, Feb 2010.

Authors 
Ken Watkins, President of Polymer Aging Concepts, Inc., has served as Principal Investigator in five condition monitoring sensor projects for electrical insulation and solid rocket propellants sponsored by the U. S. Department of Energy and the U. S. Department of Defense. He graduated from Georgia Tech in Mechanical Engineering, and is a registered professional engineer in the State of Georgia and a U. S. Patent Agent.

C. P Wong is Regent's Professor in the School of Materials Science and Engineering at The Georgia Institute of Technology in Atlanta, GA. He graduated with a Bachelors of Science degree at Purdue University and a Ph.

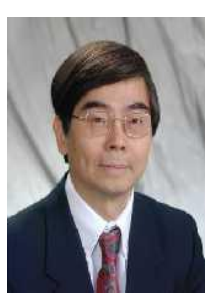
D. At Pennsylvania State University. Dr. Wong served as an ATT Bell Laboratory Fellow, and has authored numerous peer-reviewed papers on conductive composites, polymeric degradation mechanisms, and electronic packaging. 